\title{
A NONHOMOGENEOUS EIGENFUNCTION EXPANSION
} BY

ALLAN M. KRALL

Non-selfadjoint expressions of the form $l y=-y^{\prime \prime}+q(x) y$ have begun to be studied extensively in the past few years, and in two instances Naimark [4] (on the interval $[0, \infty)$ ) and Kemp [3] (on the interval $(-\infty, \infty)$ ) have achieved expansions in terms of eigenfunctions of $l$. By applying a suitable boundary condition, this paper generates an ordinary expansion and also a nonhomogeneous expansion, that is, an expansion involving solutions of $-y^{\prime \prime}+(q(x)-\lambda) y=K(x)$, in $[0, \infty)$, thus extending the work of Naimark [4].

Nonhomogeneous expansions originated with Hilb [2] and Betschler [1].

1. The operator $L$. We consider a differential expression of the form $l y=-y^{\prime \prime}+q(x) y, 0 \leqq x<\infty$, where $q(x)$ is an arbitrary measurable complex function satisfying $\int_{0}^{\infty}|q(x)| d x<\infty$.

We denote by $D_{0}$ those functions $f$ defined on $[0, \infty)$ and satisfying

1. $f$ is in $L^{2}(0, \infty)$,

2. $f^{\prime}$ exists and is absolutely continuous on every finite subinterval of $[0, \infty)$,

3. If is in $L^{2}(0, \infty)$.

Let $K(x)$ be an arbitrary complex-valued function in $L^{2}(0, \infty)$, and let $\alpha$ and $\beta$ be arbitrary complex numbers. We denote by $D$ those functions $f$ satisfying

1. $f$ is in $D_{0}$,

2. $\int_{0}^{\infty} K(x) f(x) d x-\beta f(0)+\alpha f^{\prime}(0)=0$.

We define the operator $L$ by $L f=$ lf for all $f$ in $D$.

THeOREM 1.1. If $|\alpha|^{2}+|\beta|^{2} \neq 0, D$ is dense in $L^{2}(0, \infty)$.

Let $U$ be those functions $g$ in $D_{0}$ vanishing with their derivatives for large $x$ and in a neighborhood of the origin and satisfying $\int_{0}^{\infty} K(x) g(x) d x=0$. Since $U$ is dense in $R^{\perp}$ (see [4, pp. 105-108]) and $U \subset D$, we see that $D$ is dense in $R^{\perp}$. If $D$ is not dense in $L^{2}(0, \infty)$, there is a nonzero vector orthogonal to $D$ and hence to $R^{\perp}$. This vector must be a scalar multiple of $R$. But then

$$
\int_{0}^{\infty} K(x) f(x) d x=0
$$

for all $f$ in $D$, and hence $\beta f(0)-\alpha f^{\prime}(0)=0$ for all $f$ in $D$. Clearly this is impossible.

2. Solutions of $l y-\lambda y=0$. Since we are working with the interval $[0, \infty)$, it is convenient to use the same solutions of $l y-\lambda y=0$ as [4].

Received by the editors October 4, 1963. 
Let $s=\lambda^{1 / 2}$ such that $0 \leqq \arg s \leqq \pi$ and $s=\sigma+i \tau$ with $\sigma$ and $\tau$ real. We will use the solutions $y_{1}(x, s)$ and $y_{2}(x, s)\left(y_{2}(x, s)=y_{1}(x,-s)\right)$ of Naimark [4, pp. 115-119] which have the following properties: $y_{1}(x, s)$ and $y_{2}(x, s)$ are jointly continuous in $x, s$ for all $x>0, \tau \geqq 0, s \neq 0$ and holomorphic in $s$ for all $\tau>0$.

$$
\begin{array}{ll}
y_{1}(x, s)=e^{i s x}(1+o(1)), & y_{1}^{\prime}(x, s)=e^{i s x}(i s+o(1)), \\
y_{2}(x, s)=e^{-i s x}(1+o(1)), & y_{2}^{\prime}(x, s)=e^{-i s x}(-i s+o(1))
\end{array}
$$

as $x \rightarrow \infty$ for all $\tau \geqq 0, s \neq 0$.

$$
\begin{aligned}
& y_{1}(x, s)=e^{i s x}\left(1+O\left(\frac{1}{s}\right)\right), \quad y_{1}^{\prime}(x, s)=i s e^{i s x}\left(1+O\left(\frac{1}{s}\right)\right), \\
& y_{2}(x, s)=e^{-i s x}\left(1+O\left(\frac{1}{s}\right)\right), \quad y_{2}^{\prime}(x, s)=-i s e^{-i s x}\left(1+O\left(\frac{1}{s}\right)\right)
\end{aligned}
$$

as $|s| \rightarrow \infty$ for all $x$ and $\tau \geqq 0$. Further

$$
W\left[y_{1}, y_{2}\right](x)=y_{1}(x, s) y_{2}^{\prime}(x, s)-y_{1}^{\prime}(x, s) y_{2}(x, s)=-2 i s .
$$

This is not exactly the way Naimark presented them. A certain amount of "welding" is necessary to put the solutions in this form.

In addition to assuming that $\int_{0}^{\infty}|q(x)| d x<\infty$, we will assume that $y_{1}(x, s)$ and $y_{2}(x, s)$ are continuous at $s=0$. This is certainly true if $\int_{0}^{\infty} e^{e x}|q(x)| d x<\infty$ for some $\varepsilon>0$ (see [4, pp. 120-125]).

\section{The spectrum of $L$.}

THEOREM 2. The operator $L$ does not have eigenvalues on the positive semiaxis $\lambda>0$. See $[4$, p. 126].

If $\lambda$ is not on the positive real axis, then $y_{1}(x, s)$ is in $L^{2}(0, \infty)$ and $y_{2}(x, s)$ is not. Hence

THEOREM 3.1. The eigenvalues of $L$ are given by solutions $s$ in the upper half plane of $N(s)=\int_{0}^{\infty} K(x) y_{1}(x, s) d x-\beta y_{1}(0, s)+\alpha y_{1}^{\prime}(0, s)=0$. The eigenvalues are then $\lambda=s^{2}$. They are finite or countable in number and accumulate only on the semiaxis $\lambda \geqq 0$.

This follows immediately since $\int_{0}^{\infty} K(x) y_{1}(x, s) d x$ is holomorphic for $\tau>0$ as are $y_{1}(0, s)$ and $y_{1}^{\prime}(0, s)$.

THEOREM 3.2. If $\alpha \neq 0$ and $K$ is in $L^{1}(0, \infty) \cap L^{2}(0, \infty)$, then the eigenvalues form a bounded set.

As $|s| \rightarrow \infty, \int_{0}^{\infty} K(x) y_{1}(x, s) d x$ is bounded as is $y_{1}(0, s)$. Since $y_{1}^{\prime}(0, s)=s(1+O(1))$ it increases without bound. Thus $N(s)$ ultimately cannot be zero. 
THEOREM 3.3. If $\alpha \neq 0, K$ is in $L^{1}(0, \infty) \cap L^{2}(0, \infty), y_{1}(x, s)$ and $y_{2}^{\prime}(x, s)$ are continuous at $s=0$ for all $x, N(s) \neq 0$ for all reals, then the number of eigenvalues of $L$ is finite.

If there were an infinite number of eigenvalues they would have to accumulate on the positive semiaxis $\lambda \geqq 0$, and then $N(s)=0$ at the accumulation point.

We will assume from now on that $K$ is in $L^{1}(0, \infty) \cap L^{2}(0, \infty)$, that $N(s) \neq 0$ for all real $s$, and finally $\alpha \neq 0$. Later we will consider the possibility of $N(s)$ vanishing for real $s$.

4. The resolvent of $L$. By variation of parameters we find the Green's function $V(\lambda, x, \xi)$ and thus the resolvent of $L$. Let

$$
\begin{aligned}
u(x, s)=-\frac{1}{2 i s}\left[y_{2}(x, s) \int_{x}^{\infty} K(z) y_{1}(z, s) d z\right. & +y_{1}(x, s) \int_{0}^{x} K(z) y_{2}(z, s) d z \\
& \left.+\left(\alpha y_{2}^{\prime}(0, s)-\beta y_{2}(0, s)\right) y_{1}(x, s)\right] .
\end{aligned}
$$

Let

$$
V_{1}(\lambda, x, \xi)=-u(\xi, s) y_{1}(x, s) / N(s)
$$

Let

$$
V_{2}(\lambda, x, \xi)= \begin{cases}-\frac{1}{2 i s} y_{1}(\xi, s) y_{2}(x, s) & \text { for } \xi>x \\ -\frac{1}{2 i s} y_{2}(\xi, s) y_{1}(x, s) & \text { for } \xi<x\end{cases}
$$

Then $V(\lambda, x, \xi)=V_{1}(\lambda, x, \xi)+V_{2}(\lambda, x, \xi)$.

THEOREM 4.1. With the exception of points lying on the semiaxis $\lambda \geqq 0$, the spectrum of $L$ consists of a finite or countable number of eigenvalues whose accumulation points lie on the semiaxis $\lambda \geqq 0$. For all remaining points not lying on the semiaxis $\lambda \geqq 0$, the resolvent is a bounded integral operator with kernel $V(\lambda, x, \xi)$ satisfying

$$
\sup _{\|f\|=1}\left\|\int_{0}^{\infty} V(\lambda, x, \xi) f(\xi) d \xi\right\| \leqq c / \tau|s| .
$$

From Naimark $\left[4\right.$, p. 131], $\sup _{\|f\|=1}\left\|\int_{0}^{\infty} V_{2}(\lambda, x, \xi) f(\xi) d \xi\right\| \leqq c_{3} / \tau|s|$. By use of the Minkowski and Schwarz inequalities we find a similar result for $V_{1}(\lambda, x, \xi)$. It is easy to see that if $f$ is in $L^{2}(0, \infty)$, then $y=R_{\lambda} f=\int_{0}^{\infty} V(\lambda, x, \xi) f(\xi) d \xi$ satisfies $\int_{0}^{\infty} K(x) y(x) d x-\beta y(0)+\alpha y^{\prime}(0)=0$ and $(l-\lambda) y=f$.

THEOREM 4.2. L is a closed operator. See $[4, p .132]$.

THEOREM 4.3. Every point of the semiaxis $\lambda \geqq 0$ is a point of the spectrum of $L$. 
Let $\lambda$ be a point not on the positive semiaxis and not an eigenvalue, and let $a$ be a positive number.

Let

$$
f(x, s)=\left\{\begin{array}{l}
\bar{y}_{2}(x, s)-\bar{u}(x, s) \int_{0}^{a} \bar{y}_{2}(z, s) u(z, s) d z / \int_{0}^{a}|u(z, s)|^{2} d z, \quad x \leqq a, \\
0, \quad x>a .
\end{array}\right.
$$

Then $\int_{0}^{a} f(x, s) u(x, s) d x=0$ and

$$
\int_{0}^{a}|f(x, s)|^{2} d x=\int_{0}^{a} f(x, s) y_{2}(x, s) d x .
$$

Thus we find for $x>a, R_{\lambda} f(x, s)=-(1 / 2 i s) y_{1}(x, s) \int_{0}^{a}|f(x, s)|^{2} d x$. Hence

$$
\left\|R_{\lambda}\right\|^{2} \geqq \frac{1}{4|s|^{2}} \int_{0}^{a}|f(x, s)|^{2} d x \int_{a}^{\infty}\left|y_{1}(x, s)\right|^{2} d x .
$$

Now choose $a$ large enough so that in $y_{1}(x, s)=e^{i s x}(1+o(1))|o(1)|<1 / 2$. Then since on any rectangle in the upper half plane with one side on the positive $s$-axis $\int_{0}^{a}|f(x, s)|^{2} d x$ is bounded away from zero we find $\left\|R_{\lambda}\right\|^{2} \geqq A e^{-2 \tau a} / 32|s|^{2} \tau$ where $A$ is a constant. Hence as $s$ approaches any point on the real axis, $\left\|R_{\lambda}\right\|$ is unbounded and the square of the point is in the spectrum of $L$.

TheOREM 4.4. Every point of the positive semiaxis $\lambda>0$ is in the continuous spectrum of $L$.

To prove this we resort to a series of lemmas.

LEMMA 4.1. If $f$ is in $D_{0}$, then $\operatorname{limit}_{x \rightarrow \infty} f(x)=0$, $\operatorname{limit}_{x \rightarrow \infty} f^{\prime}(x)=0$. See $[4, p .132]$.

Lemma 4.2. If $g$ is in the domain of $L^{*}$, then $\bar{g}$ is in $D_{0}$.

If $\lambda$ is not in the spectrum, we have $f(x)=\int_{0}^{\infty} V(\lambda, x, \xi)(L-\lambda) f(\xi) d \xi$ for all $f$ in $D$.

If $g$ is in the domain of $L^{*}$,

$$
\begin{aligned}
\int_{0}^{\infty}[(L-\lambda) f(\xi)] \bar{g}(\xi) d \xi & =([L-\lambda] f, g)=\left(f,\left[L^{*}-\lambda\right] g\right) \\
& =\int_{0}^{\infty} \int_{0}^{\infty} V(\lambda, x, \xi)[(L-\lambda) f(\xi)] \overline{\left[\left(L^{*}-\lambda\right) g(x)\right]} d \xi d x \\
& =\int_{0}^{\infty}[(L-\lambda) f(\xi)] \overline{\left(\int_{0}^{\infty} \nabla(\lambda, x, \xi)\left[\left(L^{*}-\lambda\right) g(x)\right] d x\right)} d \xi .
\end{aligned}
$$


Since $(L-\lambda) f$ may be arbitrary in $L^{2}(0, \infty)$, we have

$$
\bar{g}(\xi)=\int_{0}^{\infty} V(\lambda, x, \xi) \overline{\left[\left(L^{*}-\bar{\lambda}\right) g(x)\right]} d x .
$$

Note that $\bar{g}(0)$ and $\bar{g}^{\prime}(0)$ exist and are finite, further that $l-\lambda$ may be applied. In doing so we find $(l-\lambda) \bar{g}$ is in $L^{2}(0, \infty)$ and $\bar{g}$ is in $D_{0}$.

LEMMA 4.3. If $f$ is in $D$ and $g$ is in the domain of $L^{*}$, then $\left(f, L^{*} g\right)=(f, l g)$ $-W[f, \bar{g}](0)$.

Integration by parts twice produces $\left(f, L^{*} g\right)=(L f, g)=(f, \overline{l g})+\left.W[f, \bar{g}](x)\right|_{0} ^{\infty}$. The upper limit is zero by Lemmas 4.1 and 4.2.

LEMMA 4.4. For every $\lambda \geqq 0$ if $g$ is orthogonal to the range of $L-\lambda$, then $(l-\lambda) \bar{g}=\rho K$ where $\rho$ is an appropriate constant.

Consider $f$ in $U$ of Theorem 1.1. By Lemma 4.3, $([L-\lambda] f, g)=(f,[l-\bar{l}] g)=0$. Since $U$ is dense in $R^{\perp},[l-\lambda] \bar{g}=\rho K$.

LEMMA 4.5. If $g$ is orthogonal to the range of $L-\lambda$ for some real $\lambda \geqq 0$, then $\bar{g}(0)=\rho \alpha$ and $\bar{g}^{\prime}(0)=\rho \beta$ where $\rho$ is defined in Lemma 4.4.

If $f$ is in $D, 0=([L-\lambda] f, g)=(f,[l-\bar{\lambda}] g)-f(0) \bar{g}^{\prime}(0)+f^{\prime}(0) \bar{g}(0)$. So by Lemma 4.4,

$$
\rho \int_{0}^{\infty} K(x) f(x) d x=f(0) \bar{g}^{\prime}(0)-f^{\prime}(0) \bar{g}(0)
$$

Also we have

$$
\int_{0}^{\infty} K(x) f(x) d x=\beta f(0)-\alpha f^{\prime}(0) .
$$

Solving these simultaneously and using the arbitrariness of $f$ completes the proof.

LEMMA 4.6. For every $\lambda>0$ if $g$ is orthogonal to the range of $L-\lambda$, then

$$
\begin{aligned}
\bar{g}(x)=-\frac{\rho}{2 i s}\left[N(-s) y_{1}(x, s)-N(s) y_{2}(x, s)\right. & \\
& \left.\quad-y_{1}(x, s) \int_{x}^{\infty} y_{2}(\xi, s) K(\xi) d \xi+y_{2}(x, s) \int_{x}^{\infty} y_{1}(\xi, s) K(\xi) d \xi\right] .
\end{aligned}
$$

This follows from solving the equation of Lemma 4.4 with the boundary conditions of Lemma 4.5 .

Since by Lemma $4.2, \bar{g}$ is in $D$, by Lemma $4.1, \operatorname{limit}_{x \rightarrow \infty} \bar{g}(x)=0$. Obviously the two integrals approach zero but unless $N(-s)=0$ and $N(s)=0$ the first two terms merely oscillate. Since $N(-s)$ and $N(s)$ are never zero for all real $s$, we have a contradiction completing the proof of the theorem. 
Note that if both $N(-s)$ and $N(s)$ are zero for a particular value of $s$ it is possible for $g$ to exist in $L^{2}(0, \infty)$.

5. Expansion of the Green's function. For simpliciy we assume that the zeros of $N(s)$ are simple zeros. We will use a theorem of Pollard [5] which extends the Cauchy integral and residue theorems to a closed contour which may contain the boundary of the function being considered as long as the function is continuous on that part of the boundary.

We choose as the contour $C_{R}$ in the $s$-plane the following: from $-R$ to $R$ along the real $s$-axis; from $R$ to $-R$ along a semicircle of radius $R$, center 0 , in the upper half plane. We choose $R$ large enough so that all the eigenvalues of $L$ are within the contour.

On the semicircle, $s V(\lambda, x, \xi)$ is analytic. On the line segment from $-R$ to $R$, $s V(\lambda, x, \xi)$ is continuous. We choose $s_{0}$ interior to $C_{R}$ such that $\lambda_{0}=s_{0}^{2}$ is not an eigenvalue of $L$.

\section{LEMMA 5.1 .}

$$
V_{2}\left(s_{0}^{2}, x, \xi\right)=\frac{1}{2 \pi} \int_{0}^{\infty}\left[\left(y_{1}(\xi, s) y_{2}(x, s)-y_{1}(x, s) y_{2}(\xi, s)\right) /\left(s^{2}-s_{0}^{2}\right)\right] d s .
$$

Consider $(1 / 2 \pi i) \int_{C_{R}}\left[V_{2}\left(s^{2}, x, \xi\right) /\left(s^{2}-s_{0}^{2}\right)\right] 2 s d s$, with $x<\xi$. By Pollard's theorem this is $V_{2}\left(s_{0}^{2}, x, \xi\right)$. Direct computation, letting $R \rightarrow \infty$, completes the proof. Since $V_{2}\left(s_{0}^{2}, x, \xi\right)$ is symmetric in $x$ and $\xi$, the same result is found if $x>\xi$.

LEMMA 5.2. Let $\left\{s_{k}^{2}\right\}_{1}^{n}$ be the eigenvalues of L. Then

$$
\begin{aligned}
V_{1}\left(s_{0}^{2}, x, \xi\right)= & \sum_{k=1}^{n} u\left(\xi, s_{k}\right) y_{1}\left(x, s_{k}\right) /\left(s_{k}^{2}-s_{0}^{2}\right) \int_{0}^{\infty} u\left(\xi, s_{k}\right) y_{1}\left(\xi, s_{k}\right) d \xi \\
& -\frac{1}{2 \pi i} \int_{0}^{\infty}\left[u(\xi, s) y_{1}(x, s) / N(s)\left(s^{2}-s_{0}^{2}\right)\right] 2 s d s \\
& -\frac{1}{2 \pi i} \int_{0}^{\infty}\left[u(\xi,-s) y_{2}(x, s) / N(-s)\left(s^{2}-s_{0}^{2}\right)\right] 2 s d s .
\end{aligned}
$$

We consider $(1 / 2 \pi i) \int_{C_{R}}\left[V_{1}\left(s^{2}, x, \xi\right) /\left(s^{2}-s_{0}^{2}\right)\right] 2 s d s$. As before the integral splits into three pieces with the integral around the semicircle vanishing as $R \rightarrow \infty$. The residues at $\left\{s_{k}^{2}\right\}_{1}^{n}$ can be evaluated by standard means giving the result.

ThEOREM 5.1. Let $\tilde{y}(x, s)=-2 i s\left(N(-s) y_{1}(x, s)-N(s) y_{2}(x, s)\right)$. Let

$$
\begin{aligned}
\tilde{u}(\xi, s)=(1 / 2 i s)\left[y_{1}(\xi, s) \int_{\xi}^{\infty} K(z) y_{2}(z, s) d z\right. & -y_{2}(\xi, s) \int_{\xi}^{\infty} K(z) y_{1}(z, s) d z \\
& \left.-N(-s) y_{1}(\xi, s)+N(s) y_{2}(\xi, s)\right]
\end{aligned}
$$

where $s$ is real. If $s_{0}^{2}$ is not in the spectrum of $L$, 


$$
\begin{aligned}
V\left(s_{0}^{2}, x, \xi\right)= & \sum_{k=1}^{n} u\left(\xi, s_{k}\right) y_{1}\left(x, s_{k}\right) /\left(s_{k}^{2}-s_{0}^{2}\right) \int_{0}^{\infty} u\left(\xi, s_{k}\right) y_{1}\left(\xi, s_{k}\right) d \xi \\
& -\frac{1}{2 \pi} \int_{0}^{\infty}\left[\tilde{u}(\xi, s) \tilde{y}(x, s) /\left(s^{2}-s_{0}^{2}\right) N(s) N(-s)\right] d s .
\end{aligned}
$$

This follows from Lemmas 5.1 and 5.2 by regrouping terms.

Note that the following properties hold.

(1) $\left(l-s^{2}\right) y(x, s)=0, \quad \int_{0}^{\infty} K(z) y(z, s) d z-\beta y(0, s)+\alpha y^{\prime}(0, s)=0$.

(2) $\left(l-s^{2}\right) u(x, s)=K(x), \quad u(0, s)=\alpha, \quad u^{\prime}(0, s)=\beta$.

(3) $\left(l-\lambda_{k}\right) y_{1}\left(x, s_{k}\right)=0, \quad \int_{0}^{\infty} K(z) y_{1}\left(z, s_{k}\right) d z-\beta y_{1}\left(0, s_{k}\right)+\alpha y_{1}^{\prime}\left(0, s_{k}\right)=0$.

(4) $\left(l-\lambda_{k}\right) u\left(x, s_{k}\right)=K(x), u\left(0, s_{k}\right)=\alpha, u^{\prime}\left(0, s_{k}\right)=\beta$.

(5) $\int_{0}^{\infty} u\left(\xi, s_{k}\right) y_{1}\left(\xi, s_{j}\right) d \xi=0$ if $k \neq j$.

6. Expansion of certain functions in $L^{2}(0, \infty)$. Let $D^{*}$ be those functions satisfying

1. $g$ is in $L^{1}(0, \infty)$,

2. $g^{\prime}$ exists and is absolutely continuous on every finite subinterval of $[0, \infty)$,

3. $l g$ is in $L^{1}(0, \infty)$,

4. $\int_{0}^{\infty} K(x) g(x) d x-\beta g(0)+\alpha g^{\prime}(0)=0$.

One can show that under these conditions $g$ is in $L^{2}(0, \infty), \operatorname{limit}_{x \rightarrow \infty} g(x)=0$, limit $_{x \rightarrow \infty} g^{\prime}(x)=0$ and that $D^{*}$ is dense in $L^{2}(0, \infty)$.

THEOREM 6.1. For $g$ in $D^{*}$,

$g(x)=\sum_{k=1}^{n} \alpha_{k} y_{1}\left(x, s_{k}\right) / \int_{0}^{\infty} u\left(\xi, s_{k}\right) y_{1}\left(\xi, s_{k}\right) d \xi-\frac{1}{2 \pi} \int_{0}^{\infty}[\alpha(s) y(x, s) / N(s) N(-s)] d s$,

where

$$
\alpha_{k}=\int_{0}^{\infty} g(x) u\left(x, s_{k}\right) d x, \quad \alpha(s)=\int_{0}^{\infty} g(x) \tilde{u}(x, s) d x .
$$

Let $f=l g-\lambda_{0} g$ where $\lambda_{0}$ is not in the spectrum of $L$. Let $s_{0}=\lambda_{0}^{1 / 2}$. Then $g(x)=\int_{0}^{\infty} V\left(s_{0}^{2}, x, \xi\right) f(\xi) d \xi$.

$$
\begin{aligned}
g(x)= & \sum_{k=1}^{n} y_{1}\left(x, s_{k}\right) \int_{0}^{\infty} u\left(\xi, s_{k}\right) f(\xi) d \xi /\left(s_{k}^{2}-s_{0}^{2}\right) \int_{0}^{\infty} u\left(\xi, s_{k}\right) y_{1}\left(\xi, s_{k}\right) d \xi \\
& -\frac{1}{2 \pi} \int_{0}^{\infty} f(\xi) \int_{0}^{\infty}\left[\tilde{u}(\xi, s) \tilde{y}(x, s) /\left(s^{2}-s_{0}^{2}\right) N(s) N(-s)\right] d s d \xi .
\end{aligned}
$$


In both the sum and integral we integrate by parts twice and reverse the order of integration in the integral, thus completing the proof.

7. A nonhomogeneous expansion. Note that the integral operator which takes $f$ in $L^{2}(0, \infty)$ into $\int_{0}^{\infty} V(\lambda, \xi, x) f(\xi) d \xi$ is bounded and sends $L^{2}(0, \infty)$ into $L^{2}(0, \infty)$.

Let $E^{*}$ be those functions $g$ satisfying

1. $g$ is in $L^{1}(0, \infty)$,

2. $g^{\prime}$ exists and is absolutely continuous on every finite subinterval of $[0, \infty)$,

3. $l g$ is in $L^{1}(0, \infty)$,

4. $\beta g(0)-\alpha g^{\prime}(0)=0$.

One can show that under these conditions $g$ is in $L^{2}(0, \infty), \operatorname{limit}_{x \rightarrow \infty} g(x)=0$, limit $_{x \rightarrow \infty} g^{\prime}(x)=0$ and that $E^{*}$ is dense in $L^{2}(0, \infty)$.

THEOREM 7.1. For $g$ in $E^{*}$,

$g(x)=\sum_{k=1}^{n} \beta_{k} u\left(x, s_{k}\right) / \int_{0}^{\infty} u\left(\xi, s_{k}\right) y_{1}\left(\xi, s_{k}\right) d \xi-\frac{1}{2 \pi} \int_{0}^{\infty}[\beta(s) \tilde{u}(x, s) / N(s) N(-s)] d s$,

where

$$
\beta_{k}=\int_{0}^{\infty} g(x) y_{1}\left(x, s_{k}\right) \quad d x, \quad \beta(s)=\int_{0}^{\infty} g(x) \tilde{y}(x, s) d x .
$$

Let $\left(l-\lambda_{0}\right) g=f+\rho K$ where $\lambda_{0}$ is not in the spectrum of $L, g(0)=\rho \alpha$, $g^{\prime}(0)=\rho \beta$. Combining thus with $\left(l-\lambda_{0}\right) V\left(\lambda_{0}, x, \xi\right)=0$ (considering $V\left(\lambda_{0}, x, \xi\right)$ as a function of $x$ ) we find $g(x)=\int_{0}^{\infty} V\left(\lambda_{0}, \xi, x\right) f(\xi) d \xi$. The remainder of the proof is similar to that of Theorem 6.1.

\section{Parseval's equality.}

TheOREM 8.1. If $f$ is in $D^{*}$ and $g$ is in $E^{*}$, then

$$
\begin{aligned}
& \int_{0}^{\infty} f(x) g(x) d x=\sum_{k=1}^{n} a_{k} \beta_{k} / \int_{0}^{\infty} u\left(\xi, s_{k}\right) y_{1}\left(\xi, s_{k}\right) d \xi \\
&-\frac{1}{2 \pi} \int_{0}^{\infty}[\alpha(s) \beta(s) / N(s) N(-s)] d s
\end{aligned}
$$

This follows immediately from either Theorem 6.1 or 7.1 .

9. Some extensions. There exists a two-fold extension of the preceding expansions: When $N(s)$ has a multiple zero not on the real axis, and when $N(s)$ has a zero on the real axis. The former corresponds to a multiple eigenvalue, the latter possibly to residual spectrum. We show how such possibilities affect the expansions by considering sample terms. The methods of proof are similar to those found in Naimark [4] and Kemp [3] and are not repeated here. 
If $s_{k}$ is a multiple zero of order $m$ of $N(s)$ not lying on the real axis then the term in the expansion of $V\left(s_{0}^{2}, x, \xi\right)$ due to $s_{k}$ is $\sum_{j=1}^{m} \sum_{p=0}^{m-j} c_{p+j} \sum_{l=0}^{p} y_{i}(x) u_{p-i}(\xi) /\left(s_{0}^{2}-s_{k}^{2}\right)^{j}$ where $c_{p+j}$ are appropriate coefficients and $y_{i}(x)=(1 / i !) \partial^{i} y_{1}(x, s) /\left.\partial \lambda^{i}\right|_{s=s_{k}}$, $u_{i}(\xi)=(1 / i !) \partial^{i} u(\xi, s) /\left.\partial \lambda^{i}\right|_{s=s_{k}}$. It can be shown that $\left\{y_{i}\right\}_{i=0}^{m-1}$ and $\left\{u_{i}\right\}_{i=0}^{m-1}$ are linearly independent and satisfy $\int_{0}^{\infty} K(x) y_{i}(x) d x-\beta y_{i}(0)+\alpha y_{i}^{\prime}(0)=0$, $\beta u_{i}(0)-\alpha u_{i}^{\prime}(0)=0, \quad\left(l-\lambda_{k}\right) y_{i}=y_{i-1}, \quad y_{-1}=0, \quad\left(l-\lambda_{k}\right) u_{i}=u_{i-1}, \quad u_{-1}=K$.

In the expansion of $g$ in $D^{*}$, the term due to $s_{k}$ is $-\sum_{r=1}^{m} c_{r} \sum_{i=0}^{r-1} y_{i}(x)$ $\int_{0}^{\infty} u_{r-i-1}(\xi) g(\xi) d \xi$, while if $g$ is in $E^{*}$ the term due to $s_{k}$ is $-\sum_{r=1}^{m} c_{r} \sum_{i=1}^{r-1} u_{r-i-1}(x) \int_{0}^{\infty} y_{i}(\xi) g(\xi) d \xi$. In Parseval's equality the term due to $s_{k}$ is

$$
-\sum_{r=1}^{m} c_{r} \sum_{i=0}^{r-1} \int_{0}^{\infty} f(x) u_{r-i-1}(x) d x \int_{0}^{\infty} g(x) y_{i}(x) d x
$$

when $f$ is in $D^{*}$ and $g$ is in $E^{*}$.

If $N(s)$ has a finite number of real zeros and $L$ has only a finite number of eigenvalues, an expansion is still possible. We alter the contour used in expanding $V\left(s_{0}^{2}, x, \xi\right)$ by following semicircles of radius $\rho$ in the upper half plane about each of the zeros and about the negative of each of the real zeros, we make these semicircles small enough so that they do not intersect or contain any other zeros of $N(s)$ in their interior. We consider a sample zero $\sigma_{i}$ : Let $C_{i}$ be a circle of radius $\rho$ about $\left|\sigma_{i}\right|$. Then the part of the integral from $-\rho+\left|\sigma_{i}\right|$ to $\rho+\left|\sigma_{i}\right|$ in the expansion of $V\left(s_{0}^{2}, x, \xi\right)$ is replaced by

$$
\frac{1}{2 \pi i} \int_{C_{i}}\left[W\left(s^{2}, x, \xi\right) /\left(s^{2}-s_{0}^{2}\right)\right] 2 s d s
$$

where integration is in a clockwise manner,

$$
W\left(s^{2}, x, \xi\right)= \begin{cases}V\left(s^{2}, x, \xi\right), & \tau \geqq 0, \\ V\left((-s)^{2}, x, \xi\right), & \tau<0 .\end{cases}
$$

In the expansion of $g$ in $D^{*}$, this becomes

$$
\frac{1}{2 \pi i} \int_{c_{i}}\left[\int_{0}^{\infty} W\left(s^{2}, x, \xi\right) g(\xi) d \xi\right] 2 s d s
$$

If $g$ is in $E^{*}$,

$$
\frac{1}{2 \pi i} \int_{c_{i}}\left[\int_{0}^{\infty} W\left(s^{2}, \xi, x\right) g(\xi) d \xi\right] 2 s d s
$$

With $f$ in $D^{*}, g$ in $E^{*}$ in the Parseval Equality this becomes

$$
\frac{1}{2 \pi i} \int_{c_{i}}\left[\int_{0}^{\infty} \int_{0}^{\infty} W\left(s^{2}, x, \xi\right) f(\xi) g(x) d \xi d x\right] 2 s d s .
$$


Note that $s=0$ can be handled in this manner if $y_{1}(x, s)$ and $y_{2}(x, s)$ are not continuous there. In this case, however, $C_{i}$ is a semicircle in the right half plane.

\section{REFERENCES}

1. Friedrich Betschler, Über Integraldarstellungen welche aus speziellen Randwert problemen bei gewohnlichen linearen inhomogenen Differentialgleichungen entspringen, Doctoral Dissertation, Julius-Maximilians-Universität, Würzburg, Germany, 1912.

2. Emil Hilb, Uber Reihentwicklungen, welche aus speziellen Randwert problemen bei gewohnlichen linearen inhomogenen Differentialgleichungen entspringen, J. Reine Angew. Math. 140 (1911), 205-229.

3. R. R. D. Kemp, A singular boundary value problem for a non-self-adjoint differential operator, Canad. J. Math. 10 (1958), 447-462.

4. M. A. Naimark, Investigation of the spectrum and expansion in eigenfunctions of a nonselfadjoint differential operator of second order on a semi-axis, Trudy Moskov. Mat. Obšč. 3 (1954), 181-270; Amer. Math. Soc. Transl. (2) 16 (1960), 103-194.

5. S. Pollard. On the conditions of Cauchy's theorem, Proc. London Math. Soc. 21 (1923), 456-482.

The Pennsylvania State University, University Park, Pennsylvania 